\title{
Diez años del programa de oxigenación por membrana extracorpórea neonatal respiratorio en un hospital público de la Argentina
} Ten years of the neonatal respiratory extracorporeal membrane oxygenation program in an Argentinian public hospital

\author{
Dra. Gisela L. Salas ${ }^{a}$, Dra. María S. Arbio ${ }^{a}$, Lic. Sonia B. Rodas ${ }^{a}$, \\ Dr. Eduardo D. Domingueza, Dr. Gustavo S. Goldsmit y Dra. Diana M. Fariña ${ }^{a}$
}

\section{RESUMEN}

La oxigenación por membrana extracorpórea (extracorporeal membrane oxygenation; ECMO, por sus siglas en inglés) es una terapia de rescate muy invasiva, para pacientes críticamente enfermos. El objetivo es mostrar los resultados de los pacientes tratados con ECMO en el Área de Terapia Intensiva Neonatal del Hospital de Pediatría Juan P. Garrahan, en los primeros diez años del programa, y analizar los factores de riesgo asociados a mortalidad en ECMO.

Método. Estudio descriptivo, retrospectivo, con análisis de factores de riesgo. Se incluyeron todos los pacientes tratados con ECMO desde abril de 2008 a febrero de 2019.

Resultados. Ingresaron 77 pacientes, 72 recién nacidos y 5 pacientes pediátricos, menores de 1 año. La mediana de edad al ingresar fue de 2 días (1-150). Peso:3200 g \pm 730 ; edad gestacional: 37,5 semanas $\pm 4,2$; el $53 \%$, del sexo masculino, y el $50 \%$, nacidos por cesárea. Los diagnósticos más frecuentes fueron hernia diafragmática congénita y síndrome de aspiración meconial. Sobrevivieron a ECMO 56 pacientes (el $73 \%$ ); de estos, fallecieron antes del alta 17 (el $30 \%)$. Las variables asociadas con mortalidad en ECMO fueron la hemorragia en el sistema nervioso central ( $\mathrm{p}:<0,01)$, la necesidad de hemodiafiltración $(p:<0,01)$, el requerimiento de inotrópicos durante ECMO (p: < 0-01) y la presencia de hemorragia sin compromiso del sistema nervioso central ( $\mathrm{p}:<0-01$ ).

Conclusiones. Este estudio refleja los resultados de los primeros 10 años de ECMO respiratoria neonatal. La aparición de complicaciones aumentóla mortalidad durante el procedimiento. Palabras clave: oxigenación por membrana extracorpórea, recién nacido, insuficiencia respiratoria.

http:/ / dx.doi.org/10.5546/ aap.2020.258

Texto completo en inglés:

http: / / dx.doi.org/10.5546/ aap.2020.eng.258

Cómo citar: Salas GL, Arbio MS, Rodas SB, Domínguez ED, et al. Diez años del programa de oxigenación por membrana extracorpórea neonatal respiratorio en un hospital público de la argentina. Arch Argent Pediatr 2020;118(4):258-264.

\section{INTRODUCCIÓN}

La oxigenación por membrana extracorpórea (extracorporeal membrane oxygenation; ECMO, por sus siglas en inglés) es un soporte cardiopulmonar, destinado a aquellos pacientes con falla respiratoria y / o cardíaca que presentan un riesgo de morir superior al $80 \%$ y en los cuales el tratamiento convencional de alta complejidad haya fracasado. Es una terapia de rescate altamente invasiva y costosa..$^{1-3}$

La ECMO permite que el pulmón y el corazón se mantengan en reposo hasta su recuperación. Esto lo logra a través de una adecuada entrega de oxígeno a los órganos; minimiza el daño generado por la asistencia respiratoria mecánica (ARM) y evita altas dosis de fármacos inotrópicos.

Existen dos modalidades de ECMO: venoarterial (ECMOVA) y venovenosa (ECMOVV). La primera provee soporte cardíaco y respiratorio, y la segunda, solo soporte respiratorio. La indicación de cada una de sus modalidades dependerá, fundamentalmente, del diagnóstico y el estado hemodinámico del paciente. Se ha reportado que la ECMOVV presenta un menor número de complicaciones que la ECMOVA, sobre todo, en el sistema nervioso central (SNC). ${ }^{4}$

A pesar de tratarse de una terapéutica con alta invasividad y posibilidad de complicaciones que pueden poner en riesgo la vida del paciente, su uso en las unidades neonatales es de alto impacto en la sobrevida de los pacientes graves. ${ }^{5}$ 
Los resultados dependen, principalmente, del diagnóstico de ingreso y del tiempo que el paciente requiera continuar con soporte de ECMO. ${ }^{6}$ Los diagnósticos que, a menudo, requieren ECMO en las unidades neonatales son los cuadros de hipertensión pulmonar primaria del recién nacido (HTPPRN), síndrome de aspiración de líquido amniótico meconial (SALAM), hernia diafragmática congénita (HDC), neumonía viral y bacteriana, sepsis, arritmias cardíacas, etc. . $^{67}$

Las primeras publicaciones exitosas del soporte de ECMO fueron las del Dr. $\mathrm{Hill}^{8}$ y luego Barlett ${ }^{9}$ en el año 1976. Las sucedieron numerosas publicaciones de descripción de casos y comparaciones con controles históricos que demostraron progresivos aumentos de sobrevida. ${ }^{10,11}$ En la década de los ochenta, se realizaron estudios controlados prospectivos que contribuyeron a la difusión de esta técnica. ${ }^{12,13}$ En 1996, se publicó el "UK Collaborative ECMO Trial", que confirmó que el tratamiento con ECMO reducía de modo significativo la mortalidad en los recién nacidos (RN) críticamente enfermos en comparación con el tratamiento convencional (mortalidad del $32 \%$ versus el $59 \%$; riesgo relativo-RR-0,55; intervalo de confianza -ICdel $95 \%$ : 0,36-0,8). ${ }^{14}$ Posteriormente, se publicó el resultado del seguimiento a largo plazo de estos pacientes, que fue alentador. ${ }^{15}$

El Programa de ECMO de la Unidad de Cuidados Intensivos Neonatales (UCIN) del Hospital de Pediatría Juan P. Garrahan comenzó en 1998 con la realización de talleres teóricoprácticos multidisciplinarios y procedimientos de ECMO en animales de experimentación (se utilizaron porcinos de la raza landrace). ${ }^{16}$ Luego de una fase de entrenamiento intensivo, se inició la etapa clínica. En el año 2008, se practicó ECMO al primer paciente neonatal en la UCIN. El Programa de ECMO del Hospital Garrahan desarrolla esta terapéutica en tres unidades diferentes: Terapia Intensiva Neonatal, Terapia Intensiva Pediátrica y Terapia de Recuperación Cardiovascular. Hasta la actualidad, más de 180 pacientes ingresaron al programa. Es miembro activo de la Extracorporeal Life Support Organization (ELSO), desde el año 2013. Cuenta con cursos de capacitación y entrenamiento de los integrantes del equipo, que incluyen talleres de Simulación de Alta Fidelidad.

El objetivo de este estudio es mostrar los resultados de los pacientes tratados con ECMO en el Área de Terapia Intensiva Neonatal, en los primeros diez años del programa, y analizar los factores de riesgo asociados a mortalidad en ECMO.

\section{MATERIAL Y MÉTODOS}

Se llevó a cabo un estudio descriptivo, retrospectivo, con análisis de factores de riesgo. Se incluyeron todos los pacientes tratados con ECMO desde abril de 2008 hasta febrero de 2019 en el Área de Neonatología del Hospital de Pediatría Juan P. Garrahan. Los criterios de inclusión al tratamiento de ECMO figuran en la Tabla 1, basados en las guías de tratamiento de la ELSO. ${ }^{17}$

En todos los casos, se solicitó la firma del consentimiento informado a los padres para la utilización anónima de los datos. El estudio fue aprobado por el Comité de Ética del hospital en el que fue realizado.

TABLA 1. Criterios de inclusión para oxigenación por membrana extracorpórea respiratoria neonatal

\section{Criterios de inclusión}

- Edad gestacional > 34 semanas.

- Asistencia respiratoria no más de 14 días (criterio relativo).

- Ausencia de enfermedades graves: anomalías cromosómicas, anomalías anatómicas o síndromes malformativos graves.

- Ausencia de HIV / PV grado II o >. Sin evidencia de daño cerebral grave.

- Ausencia de sangrado masivo.

- Presencia de insuficiencia respiratoria grave:

- $\quad \mathrm{IO}^{*}>40$, en tres determinaciones arteriales, en un período de 3-5 $\mathrm{h}\left(\mathrm{IO}=\mathrm{MAP} \times \mathrm{FiO}_{2} / \mathrm{PaO}_{2}\right.$ posductal).

- Descompensación grave con $\mathrm{PaO}_{2}<40$ torr, sin respuesta a la terapia máxima (ventilación de alta frecuencia, administración de óxido nítrico inhalado).

- Episodios de descompensación, tres o más, durante 12 h, que requieren reanimación con presión positiva.

- Disfunción cardíaca grave, sin respuesta a terapia convencional:

- Ecocardiograma que demuestra disfunción ventricular grave con soporte vasoactivo máximo: dopamina $\geq 20 \mu \mathrm{g} / \mathrm{kg} / \mathrm{min}$; epinefrina $\geq 0,5 \mu \mathrm{g} / \mathrm{kg} / \mathrm{min}$; dobutamina $\geq 20 \mu \mathrm{g} / \mathrm{kg} / \mathrm{min}$; milrinona = $0,8 \mu \mathrm{g} / \mathrm{kg} / \mathrm{min}$. - Requerimientos continuos de expansiones de volumen.

* IO: índice de oxigenación; MAP (presión media en la vía aérea) x $\mathrm{FIO}_{2}$ (fracción inspirada de oxígeno)/ presión parcial de oxígeno; HIV-PV: hemorragia intraventricular-periventricular. 
Organización del Programa de ECMO: El equipo de ECMO estuvo formado por un coordinador médico y un coordinador de Enfermería, dieciocho médicos neonatólogos y veinticinco enfermeros neonatales. Además, cirujanos cardiovasculares e integrantes de los Servicios de Hematología, Hemoterapia, Cardiología, Farmacia y Diagnóstico por Imágenes. La decisión del ingreso y egreso del paciente a $E C M O$ era consensuada entre el equipo de $E C M O$ y el equipo médico a cargo del paciente. En todos los casos, se requirió el consentimiento informado de los padres para su ingreso. ${ }^{16}$

Protocolo de manejo del paciente durante la ECMO: El paciente se colocó en una servocuna con sistema de elevación, en decúbito dorsal, con la cabeza ubicada a los pies para la canulación de los vasos del cuello. Se colocó un monitor multiparamétrico con tensión arterial invasiva, temperatura corporal central, oximetría de pulso, registro electrocardiográfico continuo. Se agregó un monitor transcutáneo de presión parcial de dióxido de carbono, saturación parenquimatosa regional por espectroscopia cercana al infrarrojo (NIRS: near infrared sprectroscopy) y monitoreo continuo de la función cerebral con electroencefalograma de amplitud integrada (EEGai).

El purgado del circuito se realizó en forma secuencial con solución salina normal, albúmina y sangre. La canulación de los vasos, la vena yugular interna derecha y la arteria carótida derecha la realizó el equipo de Cirugía Cardiovascular. Se practicó radiografía de tórax y ecocardiograma para confirmar la posición de las cánulas.

La anticoagulación se realizó con infusión continua de heparina sódica. Esta se monitorizó, inicialmente, con el tiempo de coagulación activada (TCA) $\left(\right.$ Medtronic ${ }^{\circledR}$ ) y controles de la hemostasia -factor anti-Xa, antitrombina, dímero D, tiempo de tromboplastina parcial activado (activated partial thromboplastin time; APTT, por sus siglas en inglés), tiempo de protrombina (TP), fibrinógeno, recuento plaquetario-. También se administró concentrado de antitrombina, ácido tranexámico y hemoderivados, según necesidad, por protocolo de manejo de transfusiones en ECMO. Diariamente, se realizaron ecocardiograma, ecografías cerebral y abdominal, análisis de laboratorio y cultivos de sangre.

Equipamiento de ECMO: Se utilizó una bomba oclusiva de rodillos (Century y/o Cobe ${ }^{\circledR}$ ), oxigenador de membrana de $0,8 \mathrm{~m}^{2}$ (Avecor ${ }^{\circledR}$, Euroset $^{\circledR}, \operatorname{Medos}^{\circledR}$, Quadrox $\left.{ }^{\circledR}\right)$ y circuito con tubuladuras tipo Super Tygon de $1 / 4$ " (un cuarto de pulgada) de sección interna por 1/16" de pared $\left(\right.$ Medos $^{\circledR}$ y Medtronic $\left.{ }^{\circledR}\right)$. El circuito contaba con monitor de saturación venosa mixta $\left(\mathrm{SvO}_{2}\right)$ y hematocrito (3M-CDI-100 ${ }^{\circledR}$ ), monitor de flujo y detector de burbujas $\left(\right.$ Transonic $^{\circledR}$ ), monitoreo de presión premembrana y posmembrana (Medtronic ${ }^{\circledR}$ modelo 66000). El hemofitro utilizado fue de marca Argimed ${ }^{\circledR}$.

Variables del estudio: Se analizaron las variables demográficas sexo, edad gestacional (EG), puntaje de Apgar, vía de nacimiento (vaginal o cesárea), días de vida al ingresar a $E C M O$, peso al ingresar a $E C M O$, diagnóstico de ingreso y días de duración del tratamiento de ECMO. Las variables de resultado fueron sobrevida al tratamiento de ECMO (aquellos pacientes que sobrevivieron luego de las $72 \mathrm{~h}$ de haberse finalizado el soporte) y sobrevida al egreso hospitalario. Se reportó la presencia de complicaciones: coagulación intravascular diseminada (CID), insuficiencia renal (definida como la necesidad de hemofiltración/ hemodiafiltración durante el soporte). La presencia de hemorragia del SNC se definió como aquella hemorragia grave que motivaba la suspensión del tratamiento, y las complicaciones hemorrágicas sin compromiso del SNC se agruparon en sangrado pulmonar, abdominal y/o del sitio de inserción de las cánulas (hemorragia no $\mathrm{SNC}$ ), el requerimiento de inotrópicos en $E C M O$, la presencia de hipertensión arterial (HTA) que requería el uso de antihipertensivos, ${ }^{18}$ arritmias cardíacas, taponamiento cardíaco, que requería drenaje pericárdico y stun cardíaco, definido como una disociación electromecánica del ventrículo izquierdo. Las complicaciones mecánicas fueron ruptura o disfunción del oxigenador y/o cambios del circuito. Se registraron también complicaciones metabólicas: la presencia de hipoglucemia (glucosa en sangre $<50 \mathrm{mg} / \mathrm{dl}$ ), hiperglucemia (glucosa en sangre $>150 \mathrm{mg} / \mathrm{dl}$ ), hiponatremia (sodio en sangre < $130 \mathrm{mEq} / \mathrm{l}$ ) o hipernatremia (sodio en sangre > $150 \mathrm{mEq} / 1$ ), alcalosis ( $\mathrm{pH}$ sanguíneo > 7,4) y / o acidosis ( $\mathrm{pH}$ sanguíneo $<7,35$ ) durante la ECMO.

Análisis estadístico: Todas las variables fueron resumidas mediante estadística descriptiva de tendencia central, posición y dispersión. Para la comparación de variables numéricas, se utilizó el test no paramétrico de Wilcoxon o T test según distribución; para variables categóricas, test de 
chi $^{2}$. Se calculó odds ratio ajustado (ORa) y su IC del $95 \%$. Se ajustaron las variables de riesgo mediante regresión logística. Se realizó el test de Hosmer-Lemeshow (test posestimación) para evaluar la capacidad de ajuste y discriminación del modelo. Se consideró estadísticamente significativo un nivel de $\mathrm{p}<0,05$. El análisis estadístico se realizó con el software STATA SE 12.0, StataCorp LP (USA).

\section{RESULTADOS}

Ingresaron a nuestro estudio 77 pacientes, 72 RN y 5 pacientes pediátricos, menores de 1 año. En todos los casos, se practicó ECMOVA. En la Tabla 2, se observan las características demográficas de la población. Los diagnósticos de ingreso a ECMO más frecuentes fueron 49 pacientes con hernia diafragmática congénita (el $64 \%$ ), 10 con hipertensión pulmonar primaria, 7 con SALAM, 3 con bronquiolitis (1 paciente con neumonía por influenza H1N1 y 2 por virus sincicial respiratorio), 4 con neumonía por Bordetella pertussis y 4 pacientes con sepsis bacteriana.

De los 21 casos fallecidos en ECMO, se había decidido la suspensión de ECMO en 7 por la mala evolución clínica o la aparición de complicaciones irreversibles. Sobrevivieron al tratamiento de ECMO 56 pacientes (el $73 \%$ ) y, al egreso hospitalario, sobrevivieron 39 pacientes (el $51 \%$ ) (Figura 1).

La comparación de las variables demográficas no mostró diferencias significativas entre el grupo de sobrevivientes y el grupo de fallecidos. En cambio, la aparición de complicaciones mostró diferencias estadísticamente significativas en ambos grupos (Tabla 3).

En el análisis multivariado, se ajustaron las variables predictoras, y se observó que la presencia de insuficiencia renal-ORa 22,7

TABLA 2. Características demográficas de la población

\begin{tabular}{lc}
\hline & N: 77 \\
\hline Sexo masculino & $41($ el 53 \%) \\
Cesárea & $38(\mathrm{el} \mathrm{50 \% )}$ \\
Peso* $^{*}$ & $3200 \mathrm{~g}( \pm 650 \mathrm{~g})$ \\
Edad gestacional $^{*}$ & 37,6 semanas $(\mathrm{DE} 3,5)$ \\
Puntaje de Apgar 5** $^{*}$ & $8( \pm 2)$ \\
Edad al ingresar** $_{\text {Días de ECMO** }}^{* *}$ & $2(1-150)$ \\
\hline
\end{tabular}

Los valores se expresan en porcentajes para las variables categóricas, * media con desvío estándar (DE) y

** mediana con rango intercuartilo.

ECMO: oxigenación por membrana extracorpórea.
(IC95\%: 1,22-420)-, la presencia de complicaciones hemorrágicas que no incluían el SNC -ORa 16,7 (IC95\%: 2,6-105)-, la aparición de hemorragia en el SNC -ORa 4,09 (IC95\%: 1,65-10,12)- y el requerimiento de inotrópicos durante la ECMO -ORa 10,03 (IC95\%: 1,5-65,4)- fueron factores de riesgo de mortalidad $(p=<0,01)$. Se observaron IC del $95 \%$ amplios, debido a la poca frecuencia de aparición de los eventos analizados, excepto en la variable de hemorragia en el SNC. Este modelo de regresión logística resultó robusto y con buena capacidad de discriminación, test de HosmerLemeshow $\mathrm{p}=0,33$ y un área bajo la curva (area under curve; $A U C$, por sus siglas en inglés) $=0,95$ (Tabla 4).

\section{DISCUSIÓN}

En este estudio, los pacientes con HDC que requirieron ECMO mostraron una sobrevida a las 72 horas de ECMO mayor que la sobrevida al egreso hospitalario. Se considera que la mortalidad dentro de las $72 \mathrm{~h}$ del tratamiento tiene mayor relación con los resultados del tratamiento de $E C M O$; en cambio, la mortalidad al momento del alta estaría mayormente relacionada con la enfermedad de base.

Nuestros resultados coinciden con las estadísticas mundiales. Según registros internacionales, la mortalidad de la HDC, que requiere $E C M O$, se ha mantenido estable en los últimos 20 años, a pesar de los avances tecnológicos. ${ }^{18}$ La ECMO pareciera ser una

FIGURA 1. Flujograma que muestra los resultados de sobrevida de los pacientes tratados con oxigenación por membrana extracorpórea

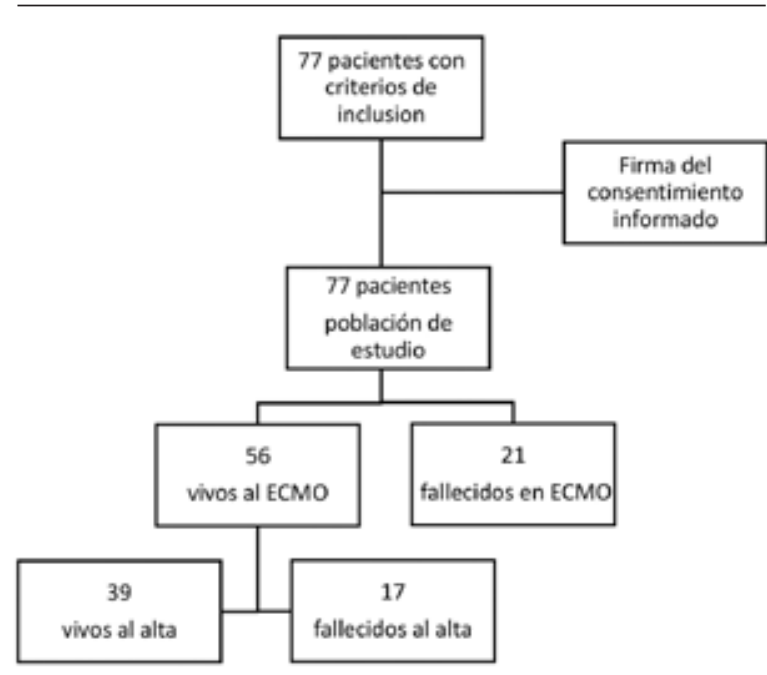

ECMO: oxigenación por membrana extracorpórea. 
terapéutica útil para estabilizar al paciente con HDC en el período prequirúrgico. Por lo tanto, poder discriminar a aquellos pacientes con hipoplasia pulmonar irreversible en esta población permitirá hacer un uso más racional de la ECMO..$^{19,20}$

Los pacientes con diagnósticos de insuficiencia respiratoria secundaria a SALAM e HTPPRN que reciben $E C M O$ presentan la mejor sobrevida en los reportes internacionales, cercana al $95 \%$.
En el otro extremo, los pacientes con HDC presentan la más baja sobrevida en ECMO por causa respiratoria en el período neonatal. Es importante destacar que, a medida que los cuidados intensivos neonatales mejoran, el ingreso a ECMO de los pacientes con SALAM e HTPPRN ha disminuido en los últimos años. La población de HDC se ha convertido en la principal causa de ingreso a ECMO en la UCIN. ${ }^{21}$

La aparición de hemorragia en el SNC fue un

TABLA 3. Comparación de variables demográficas y complicaciones según la mortalidad

\begin{tabular}{|c|c|c|c|}
\hline & Vivos, $n=56$ & Muertos, $\mathrm{n}=21$ & $p$ \\
\hline $\mathrm{EG}(\mathrm{sem} .)^{*}$ & $37,7( \pm 4,14)$ & $37,3( \pm 1,2)$ & 0,7 \\
\hline Sexo masculino & $30 / 56$ & $11 / 21$ & 0,92 \\
\hline $\operatorname{Peso}(g)^{*}$ & $3250( \pm 676)$ & $3100( \pm 550)$ & 0,34 \\
\hline Apgar 5 min* & $7,7( \pm 1,6)$ & $8,1( \pm 1,7)$ & 0,34 \\
\hline Vía de nac.: cesárea** & $27 / 56$ & $12 / 21$ & 0,48 \\
\hline Edad (días) ${ }^{* *}$ & 8,5 & 8,9 & 0,9 \\
\hline Días de ECMO** & $7,46( \pm 7,2)$ & $5,3( \pm 5,3)$ & 0,22 \\
\hline $\begin{aligned} \text { Diagnósticos: } & \\
- & \text { HDC } \\
- & \text { HTPPRN } \\
- & \text { SALAM } \\
- & \text { Bronquiolitis } \\
- & \text { Sepsis } \\
- & \text { Bordetella pertussis }\end{aligned}$ & $\begin{array}{c}35 / 49 \\
8 / 10 \\
7 / 7 \\
2 / 3 \\
2 / 4 \\
2 / 4\end{array}$ & $\begin{array}{c}14 / 49 \\
2 / 10 \\
0 \\
1 / 3 \\
2 / 4 \\
2 / 4\end{array}$ & 0,61 \\
\hline Insuficiencia renal & $1 / 3$ & $2 / 3$ & $<0,01$ \\
\hline Hemorragia no SNC & $3 / 11$ & $8 / 11$ & $<0,01$ \\
\hline Hemorragia SNC & $8 / 21$ & $13 / 21$ & $<0,01$ \\
\hline Req. de inotrópicos & $3 / 12$ & $9 / 12$ & $<0,01$ \\
\hline Complicaciones mecánicas & $12 / 15$ & $3 / 15$ & 0,46 \\
\hline CID & $5 / 11$ & $6 / 11$ & 0,28 \\
\hline HTA & $7 / 11$ & $4 / 11$ & 0,46 \\
\hline Arritmias & $2 / 2$ & 0 & 0,38 \\
\hline Stun cardíaco & $1 / 2$ & $1 / 2$ & 0,46 \\
\hline Taponamiento cardíaco & $6 / 9$ & $3 / 9$ & 0,16 \\
\hline
\end{tabular}

Los resultados se expresan en fracciones: número/ total de pacientes. * Media y desvío estándar (DE).

** Mediana y rango intercuartilo (RIQ).

EG: edad gestacional; ECMO: oxigenación por membrana extracorpórea; HDC: hernia diafragmática congénita; HTPPRN: hipertensión pulmonar primaria del recién nacido; SALAM: síndrome de aspiración meconial;

SNC: sistema nervioso central; CID: coagulación intravascular diseminada; HTA: hipertensión arterial.

TABLA 4. Análisis multivariado de las complicaciones que mostraron significancia estadística en ambos grupos

\begin{tabular}{lccccc}
\hline Complicaciones & Vivos, $\mathbf{n}=\mathbf{5 6}$ & Muertos, $\mathbf{n = 2 1}$ & $p$ & ORa & IC $\mathbf{9 5} \%$ \\
\hline Insuficiencia renal & 1 & 2 & $<0,01$ & 22,7 & {$[1,22-420\}$} \\
Hemorragia no SNC & 3 & 8 & $<0,01$ & 16,7 & {$[2,6-105]$} \\
Hemorragia SNC & 8 & 13 & $<0,01$ & 4,09 & {$[1,65-10,12]$} \\
Req. de inotrópicos & 3 & 9 & $<0,01$ & 10,03 & {$[1,5-65,4]$} \\
\hline
\end{tabular}

ORa: odds ratio ajustado; IC $95 \%$ : intervalo de confianza del $95 \%$; SNC: sistema nervioso central.

Hosmer-Lemeshow AUC: 0,95, p = 0,33. 
factor de riesgo independiente de mortalidad en esta población. En un estudio de cohorte prospectivo de 514 pacientes neonatales y pediátricos, con una sobrevida global del $55 \%$, se diagnosticó la presencia de hemorragia cerebral en el $16 \% .{ }^{22}$ En nuestro estudio, la presencia de esta complicación fue del $25 \%$. En el período inicial del programa, hubo un aumento en la incidencia de esta complicación, que ha disminuido en los últimos 2 años, en concordancia con las modificaciones en los protocolos de anticoagulación.

Se observó una incidencia de aparición de hemorragias no SNC (sangrado pulmonar, abdominal y en el sitio de inserción de las cánulas) del $16 \%$, que fue menor que la reportada por los datos de la ELSO. ${ }^{23}$ Sin embargo, la presencia de complicación fue un factor de riesgo de mortalidad en esta población $(p=<0,01)$.

Las complicaciones hemorrágicas en los pacientes que reciben ECMO producen una morbilidad y mortalidad significativa. ${ }^{24} \mathrm{Su}$ prevención requiere de una titulación cuidadosa de la terapia de anticoagulación, que minimiza el riesgo de aparición de dichas complicaciones. ${ }^{1,25}$

El requerimiento de técnicas dialíticas (hemodiafiltación o hemofiltración) durante la ECMO está condicionado, fundamentalmente, por la presencia de insuficiencia renal, oliguria $\mathrm{o}$ anuria y/o sobrecarga hídrica. En nuestro estudio, se observó que un 6,5\% de los pacientes analizados necesitaron hemodiafiltración. Esta incidencia fue menor que la reportada en los registros de la ELSO, que correspondieron a un $15,7 \% .{ }^{26,27}$ La presencia de insuficiencia renal que requería terapia dialítica en la ECMO aumentaba el riesgo de morir en esta población.

Otra de las variables asociadas a mortalidad en la $E C M O$, en este estudio, fue el requerimiento de inotrópicos durante el soporte. La ECMO ofrece soporte cardíaco parcial. Se estima que el $80 \%$ del gasto cardíaco del paciente es aportado por la ECMO, lo que permite que el gasto cardíaco nativo del paciente irrigue las arterias coronarias y posibilite la rápida mejoría de la función cardíaca. ${ }^{28}$ Se espera que, durante el inicio de ECMOVA, se suspendan medicaciones inotrópicas y vasopresoras. Cuando esto no es posible, aumenta el riesgo de muerte en la ECMO.

Si bien se trata de un reporte inédito en la Argentina, por la cantidad de pacientes neonatales y la complejidad de la terapéutica aplicada y su desarrollo en un hospital público, presenta la debilidad de informar resultados de un único centro y un limitado número de pacientes y complicaciones. Del análisis de estos primeros casos, la incidencia de dificultades y su relación con los resultados nos permitirán trazar un camino de mejora continua. Estos estarán apuntados a disminuir el número de complicaciones e incorporar nuevas tecnologías, con la finalidad de reducir la mortalidad y la morbilidad a largo plazo de los pacientes sometidos a ECMO.

\section{CONCLUSIÓN}

Este estudio refleja los resultados de los primeros 10 años de ECMO respiratoria neonatal, los cuales fueron similares a los reportes internacionales. La aparición de complicaciones durante el procedimiento aumentó la mortalidad.

\section{REFERENCIAS}

1. Van Ommen C, Neunert C, Chitlur M. Neonatal ECMO. Front Med (Lausanne). 2018; 5:289.

2. Raman L, Dalton HJ. Year in Review 2015: Extracorporeal Membrane Oxygenation. Respir Care. 2016; 61(7):986-91.

3. Petrou S, Bischof M, Bennett C, Elbourne D, et al. Costeffectiveness of neonatal extracorporeal membrane oxygenation based on 7-year results from the United Kingdom Collaborative ECMO Trial. Pediatrics. 2006; 117(5):1640-9.

4. Delaplain PT, Jancelewicz T, Di Nardo M, Zhang L, et al. Management preferences in ECMO mode for congenital diaphragmatic hernia. J Pediatr Surg. 2019; 54(5):903-8.

5. Kattan J, Godoy L, Zavala A, Faunes M, et al. Improvement of survival in infants with congenital diaphragmatichernia in recent years: effect of ECMO availability and associated factors. Pediatr Surg Int. 2010; 26(7):671-6.

6. Smith M, Vukomanovic A, Brodie D, Thiagarajan R, et al. Duration of veno-arterial extracorporeal life support (VA ECMO) and outcome: an analysis of the Extracorporeal Life Support Organization (ELSO) registry. Crit Care. 2017; 21(1):45.

7. Fletcher K, Chapman R, Keene S. An overview of medical ECMO for neonates. Semin Perinatol. 2018; 42(2):68-79.

8. Hill JD, De Leva MR, Fallat RJ, Bramson ML, et al. Acute respiratory insufficiency. Treatment with prolonged extracorporeal oxygenation. J Thorac Cardiovasc Surg. 1972; 64(4):551-62.

9. Bartlett RH, Gazzaniga AB, Jefferies MR, Huxtable RF, et al. Extracorporeal membrane oxygenation (ECMO) cardiopulmonary support in infancy. Trans Am Soc Artif Intern Organs. 1976; 22(1):80-93.

10. Bartlett RH, Andrews AF, Toomasian JM, Haiduc NJ, et al. Extracorporeal membrane oxygenation for newborn respiratory failure: forty-five cases. Surgery. 1982; 92(2):425-33.

11. Short BL, Miller MK, Anderson KD. Extracorporeal membrane oxygenation in the management of respiratory failure in the newborn. Clin Perinatol. 1987; 14(3):737-48.

12. Bartlett RH, Roloff DW, Cornell RG, Andrews AF, et al. Extracorporeal circulation in neonatal respiratory failure: a prospective randomized study. Pediatrics. 1985; 76(4): 479-87. 
13. O'Rourke PP, Crone RK, Vacanti JP, Ware JP, et al. Extracorporeal membrane oxygenation and conventional medical therapy in neonates with persistent pulmonary hypertension of the newborn: a prospective randomized study. Pediatrics. 1989; 84(6):957-63.

14. UK Collaborative ECMO Trial Group. UK collaborative randomized trial of neonatal extracorporeal membrane oxygenation. Lancet 1996; 348(9020):75-82.

15. BennetC, Johnson A, Field D, Elbourne D; UK Collaborative ECMO Trial Group. UK Collaborative randomised trial of neonatal extracorporeal membrane oxigenation: follow up to age 4 years. Lancet. 2001; 357(9262):1094-6.

16. Domínguez ED, Salas GL, Valdés M, Rubio C, et al. Oxigenación por Membrana Extracorpórea (ECMO): Experiencia en una Unidad de Cuidado Intensivo Neonatal. Arch Argent Pediatr. 2012; 110(5):404-11.

17. Gray B, Rintoul N. Guidelines for Neonatal Respiratory Failure. Extracorporeal Life Organization, Version 1.4. Ann Arbor: ELSO; 2017. [Acceso: 27 de diciembre de 2019]. Disponible en: https:/ / www.elso.org/Portals/0/ ELSOGuidelinesNeonatalRespiratoryFailurev1_4.pdf.

18. Kays DW. ECMO in CDH: Is there a role? Semin Pediatr Surg. 2017; 26(3):166-70.

19. McHoney M, Hammond P. Role of ECMO in congenital diaphragmatic hernia. Arch Dis Child Fetal Neonatal Ed. 2018; 103(2):F178-81.

20. Rambaud J, Guilbert J, Guellec I, Jean S, et al. Place de $1 \square$ assistence respiratoire et circulatoire extracorporelle de courte durée (ECMO), post-cardiotomie exclue, dans la prise en charge des défaillances graves du nouveau-né et de l'enfant. Arch Pediatr. 2017; 24(6):578-86.

21. Mugford M, ElbourneD, Field D. Extracorporeal membrane oxygenation for severe respiratory failure in newborn infants. Cochrane Database Syst Rev. 2008; (3):CD001340.

22. Dalton HJ, Reeder R, Garcia-Filion P, Holubkov R, et al. Factors associated with bleeding and thrombosis in children receiving extracorporeal membrane oxygenation. Am J Respir Crit Care Med. 2017; 196:762-71.

23. Brogan T, Lequier L, Lorusso R, McLaren G, et al (eds.). Extracorporeal Life Support: The Elso Red Book. $5^{\text {th }}$ ed. Ann Arbor, MI: ELSO; 2019.

24. Kamdar A, Rintoul N, Raffini L. Anticoagulation in neonatal ECMO. Semin Perinatol. 2018; 42(2):122-8.

25. Cashen K, Reeder R, Dalton HJ, Berg RA, et al. Functional status of neonatal and pediatric patients after extracorporeal membrane oxygenation. Pediatr Crit Care Med. 2017; 18(6):561-70.

26. Thiagarajan RR, Barbaro RP, Rycus PT, Mcmullan DM, et al. Extracorporeal Life Support Organization Registry International Report 2016. ASAIO J. 2017; 63(1):60-7.

27. Mahmood B, Newton D, Pallotto EK. Current trends in neonatal ECMO. Semin Perinatol. 2018; 42(2):80-8.

28. Sewell EK, Piazza AJ, Davis J, Heard ML, et al. Inotrope Needs in Neonates Requiring Extracorporeal Membrane Oxygenation for Respiratory Failure. J Pediatr. 2019; 214:128-33.

\section{Archivos hace 75 años}

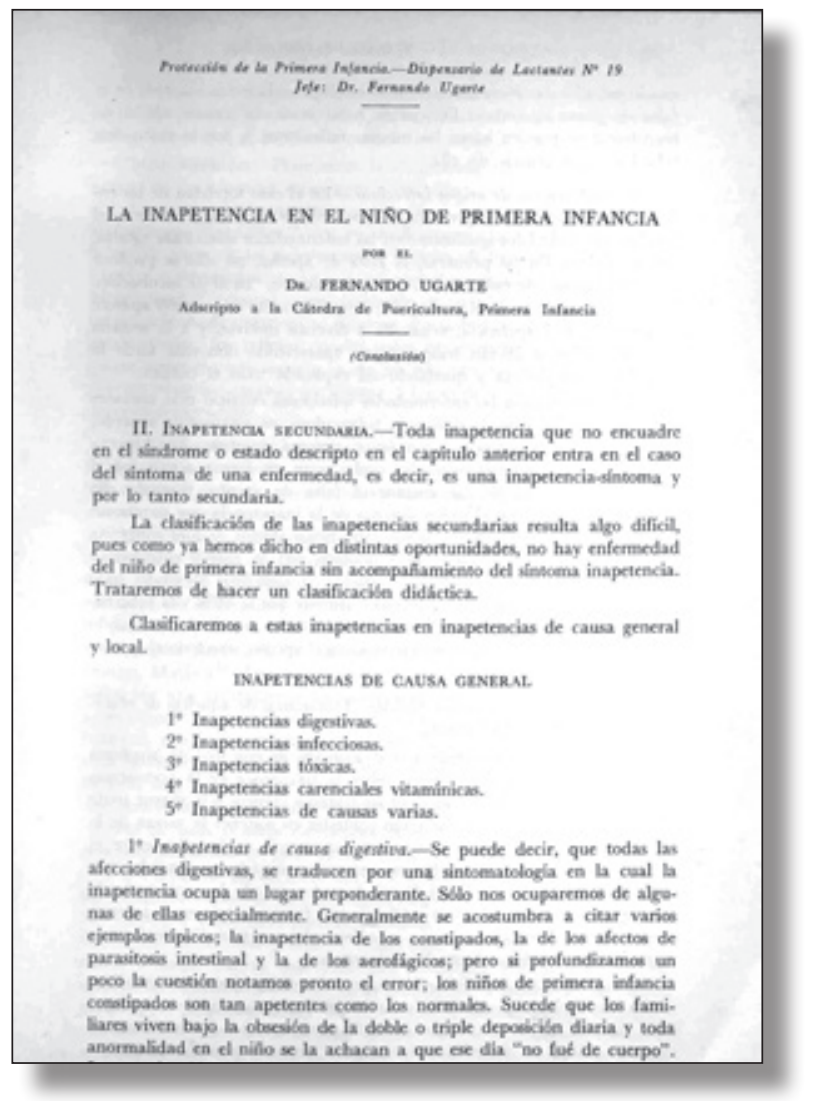

El texto completo se encuentra disponible en la versión electrónica de este número. 